\title{
Multi-user Remote Lab: Timetable Scheduling Using Simplex Nondominated Sorting Genetic Algorithm
}

\author{
SEID MIAD ZANDAVI, VERA CHUNG, and ALI ANAISSI, School of Computer Science, \\ The University of Sydney, Australia
}

\begin{abstract}
The scheduling of multi-user remote laboratories is modeled as a multimodal function for the proposed optimization algorithm. The hybrid optimization algorithm, hybridization of the Nelder-Mead Simplex algorithm, and Non-dominated Sorting Genetic Algorithm (NSGA), named Simplex Non-dominated Sorting Genetic Algorithm (SNSGA), is proposed to optimize the timetable problem for the remote laboratories to coordinate shared access. The proposed algorithm utilizes the Simplex algorithm in terms of exploration and NSGA for sorting local optimum points with consideration of potential areas. SNSGA is applied to difficult nonlinear continuous multimodal functions, and its performance is compared with hybrid Simplex Particle Swarm Optimization, Simplex Genetic Algorithm, and other heuristic algorithms. The results show that SNSGA has a competitive performance to address timetable problems.
\end{abstract}

CCS Concepts: • Theory of computation $\rightarrow$ Theory of randomized search heuristics; $\bullet$ Applied computing $\rightarrow$ Distance learning; • Mathematics of computing $\rightarrow$ Evolutionary algorithms;

Additional Key Words and Phrases: Remote laboratory, simplex algorithm, genetic algorithm, timetable problem, multimodal function

\section{ACM Reference format:}

Seid Miad Zandavi, Vera Chung, and Ali Anaissi. 2021. Multi-user Remote Lab: Timetable Scheduling Using Simplex Nondominated Sorting Genetic Algorithm. ACM/IMS Trans. Data Sci. 2, 2, Article 14 (March 2021), 13 pages.

https://doi.org/10.1145/3437260

\section{INTRODUCTION}

Every engineering student is expected to have a set of professional skills related to teamwork, oral and written communication, and impact of engineering solutions, life-long learning, and knowledge of contemporary issues [1], contributing to maintaining and improving their country's social well-being and economic prosperity. When making the transition to the labour market, graduates need not only to have a solid theoretical-practical knowledge in their field of specialization but also appropriate soft or generic skills, such as communication, teamwork, time management, problemsolving, learning aptitude, and the ability to mange stress and heavy workloads.

\footnotetext{
Authors' addresses: S. M. Zandavi, V. Chung, and A. Anaissi at the School of Computer Science, The University of Sydney, Camperdown, NSW 2006, Sydney, Australia; emails: \{miad.zandavi, vera.chung, ali.anaissi\}@sydney.edu.au.

Permission to make digital or hard copies of all or part of this work for personal or classroom use is granted without fee provided that copies are not made or distributed for profit or commercial advantage and that copies bear this notice and the full citation on the first page. Copyrights for components of this work owned by others than ACM must be honored. Abstracting with credit is permitted. To copy otherwise, or republish, to post on servers or to redistribute to lists, requires prior specific permission and/or a fee. Request permissions from permissions@acm.org.

(c) 2021 Association for Computing Machinery.

2577-3224/2021/03-ART14 \$15.00

https://doi.org/10.1145/3437260
} 
Lab experiments allow students to efficiently apply theoretical concepts to practical situations, as well as handling instruments, equipment and data. This practice contributes to build and consolidate knowledge and competences [2]. Simulation and remote labs provide an alternative and/or complementary way to develop knowledge and competences being a "blended" or "hybrid" approach to laboratory learning-a combination of hands-on labs, simulation and remote labs-the most effective [3]. These online resources offer new learning spaces and have three main advantages: accessibility, availability and safety [4]. They allow teachers to diversify their classes in a simple way; they do not have to think if the lab is free and are suitable for classes with a lot of students. Likewise, they allow students to practice at their own pace, potentiating student' autonomous work, time management and responsibility [5]. The use of these Information and Communication Technology (ICT) tools in lower levels of education (basic or secondary) are likely to appeal young students as they are a generation of digital natives. This usage may help to reduce some of the apathy and fear students feel concerning science and contribute to scaffold Science, Technology, Engineering and Mathematics (STEM) courses.

While with simulations the results obtained are from computational models, with remote labs the results are real, as a remote lab is a real lab in which the user and the equipment/instruments are physically apart. To perform an experiment the user has to access the internet and control the physical parameters of the experiment, through a computer or smartphone interface [4]. Remote labs, being tools that combine virtual access and real experimental results, have the advantages of simulations and hands-on labs.

The laboratory is an essential and supplementary part of learning to bridge the gap between theory and practice. These days, new technologies provide laboratories with a different form of observation, experimentation, and investigation whereby distance learning garnered the attention of researchers. Hence, the remote lab has been designed to perform the ubiquitous platform from faraway places for learners. The most important feature of remote laboratories is to share expensive resources across the world [2, 5]. In this regard, some benefits such as flexible access, sharing resources [3, 4], shared architecture (e.g., Sahara labs [4] and MIT's iLab [3]), security of users, data and devices [6] among many other benefits have been shown to increase attention of users. Regarding the shared platform, user allocations have been a significant issue. Thus, proper scheduling can respond to the demand for having access to the remote laboratory.

Scheduling problems are formulated as a nonlinear optimization problem. Heuristic optimization algorithms are carried out to address this problem. For example, Simulated Annealing (SA) [6], Tabu search, Genetic Algorithm (GA) [7], Ant Colony Optimization [8], and so on, have been proposed as a methodology to deal with the timetabling problem. References [9] and [10] solved course scheduling by applying Hybrid Particle Optimization and Hybrid Harmony Search, respectively. High school timetabling has been proposed as one of the hard nonlinear problems because of considering resources and events [11]. Reference [12] proposed a hybrid algorithm with a combination of ant colony optimization and Large Neighborhood Search (LNS). As an example, the LNS approach carries out the roulette wheel method based on each wealthy neighborhood to address hybrid algorithms disadvantages [13]. SA and Particle Swarm Optimization (PSO) are combined to schedule different types of trains on a single railway track [14]. Further, regarding education setting, university timetable planning has been proposed as constraint-satisfaction problems optimizing by GA $[15,16]$.

Meta-heuristic optimization algorithms fail to respond to the online scheduling problem, because not only are they designed to solve most of the optimization problems but also they do not have enough efficiency in time-consuming [17]. On this account, Nelder-Mead simplex has been successfully hybridized with the meta-heuristics to increase the rate of convergence. As an 
example, Nelder-Mead simplex Particle Swarm Optimization (NM-PSO) [18, 19], Nelder-Mead simplex Genetic Algorithm (NM-GA) [18], Stochastic Dual Simplex Algorithm (SDSA) [20], and Simplex Non-dominated Sorting Genetic Algorithm-II (simplex-NSGA-II) [21, 22] utilize the simplex algorithm as a supplementary part of the main algorithm to improve the exploitation. The main reason is to reach a better compromise between computational efforts and accuracy.

Developing an efficient optimization algorithm is investigated to make better organization when many requests occupy the shared platform. The proposed hybrid algorithm is generated to solve scheduling problems, which are modeled as multimodal functions. The proposed algorithm, named Simplex Non-dominated Sorting Genetic Algorithm (SNSGA), consists of two parts; the simplex approach for increasing exploration and NSGA as finding promising areas. When NSGA sorts local optimum points with consideration of a potential area that probably contains a global minimum, the simplex techniques provide a whole searching domain with a diverse exploration. Hence, NSGA and the simplex module have enough potential to detect promising area and increase the diversity of individuals, respectively.

The organization of this article is as follows: the remote lab timetabling problem is explained in Section 2. The utilized algorithm, the Nelder-mead simplex algorithm, and nondominated sorting genetic algorithm are detailed in Section 3. The proposed hybrid algorithm is presented in Section 4. Numerical results are made in Section 5. Finally, the conclusion is drawn in Section 6.

\section{REMOTE LAB TIMETABLING PROBLEM}

The remote lab has been organized as a ubiquitous education technology to expose a set of lab equipment kinds to the world via the internet. Accessing to the appropriate device can play a supplementary role for both education and research activities in a variety of science and engineering disciplines. To enable better usage of available expensive equipment, a remote lab service provider can host multiple users at the same time to amortize the cost of ownership as much as possible. In many situations, allocating the available lab facilities to meet the users' demand is not a straightforward decision. The main constraint is the limited number of available resources versus the possibility of abundant users' request to employ a set of lab resources.

Accordingly, the number of teacher logs per task changes considerably amongst the different implementations, regardless of implementation topic, course contents level, or type of task. It is not clear which are the factors that influence this variation, since it shows no correlations with any analysed aspect.

Teacher mediation in class and support/feedback in the proposed tasks also proves to play an important role in students' engagement with the tool and ultimately its success, which is in accordance with literature [23]. While using a new tool like remote lab platforms, teachers' enthusiasm and familiarity (teachers own usage) is crucial, not only to arouse student perception of its utility but also to stimulate their enthusiasm and will to use it, as some students may be reluctant to use it and/ or may experience some difficulties in the beginning. Teachers should be aware of it and be prepared to help students overcome these initial difficulties, quickly enough to avoid their disappointment. In fact, teachers' attention to remote labs-introduction and support during the tasks-plays a crucial role in students' engagement, as was already reported in literature [4]. This support contributes not only to students' satisfaction with the tool but also helps them to understand the usefulness of the resource, motivating its usage [24, 25].

To begin with, handling such shared access among users with different preferences, employing an optimization technique for resource allocation and scheduling strategy to satisfy a set of predefined constraints is inevitable. The remote lab timetabling problem involves assigning a set of a limited number of available resources to users' requests to optimize an objective function that reflects unused resources. Also, the constraints to be met are as follows. 
- Each user can only employ one rig across any requests.

- The amount of time that a user can employ a rig is limited. Such a limitation needs to be determined based on the nature of the experiment as well as the number of other users waiting in queue to access the same rig.

- None of the students can have inactive time (also referred to as idle time) during the possession of a rig.

The primary aim of this timetable problem is to minimize the number of unused rigs of each rig type. Such a minimization leads to increase in the total number of satisfied users (about the constraints mentioned above). Notably, the objective function of this optimization system to be minimized can be modeled as follows:

$$
f=\sum_{l=1}^{L}\left\|[X]_{u \times R}(t, l) \times\left[[C]_{R \times 1}(l)-[P]_{R \times 1}(t, l)\right]\right\|,
$$

where $L$ is the number of rig types, and $R$ is defined as a rig set in each type. $C$ and $P$ are the capacity of rig $R$ and the total number of users who are employing such a rig, respectively. $[x]_{u \times R} \in 0,1$ is a matrix that is set to one if the associated rig $R$ is applied for an experiment in each time-slot $t$ and rig type $l$, otherwise it is equal to zero.

As an example, let us assume that four users want to use a remote lab system consists of 3 rig types (i.e., $L=3$ ). It is assumed that each rig type has one rig (i.e., $R=1$ ), while each of them has three capacity levels (i.e., $C=3$ ). While each of the first three users uses the first rig type that includes the first rig, then the associated $[X(1,1)](t, l)$ is set to 1 . So, the value of $[P(1)](t, l)$ is set to 3. While the requests of forth user to access any other rig types can be delivered without any delay, any request of this user to access rig 1 must be queued by the system as other users occupy it. In such a circumstance, $[X(1,1)](t, l)$ remains to one while the value of $(C-P)$ is equal to 0 . By terminating any experiment of the first three users, rig 1 is ready to be used by the fourth user. This can be inferred from the above equation by noting that $(C-P)$ is not equal to zero anymore. Figure 1 demonstrates a schema for the scheduling process of the case mentioned above.

In Figure 1, $X=1$ represents a situation when all users select the Rig 1 in Rig Type 1. Also, Rig 1 is not allocated to the fourth user as others occupy it. When an experiment terminates at the defined procedure, the fourth user can be assigned to use Rig 1 after elapsing the threshold time ( $T=5 \mathrm{~min}$ in this example). Thus, it is imperative to use an efficient search method to produce the optimal timetable to satisfy all constraints.

\section{SIMPLEX AND NONDOMINATED SORTING GENETIC ALGORITHM}

This section represents the general setting out of the utilized algorithms in the proposed hybrid model. The Nelder-Mead Simplex Algorithm and Nondominated Sorting Genetic Algorithm are combined to improve the performance of optimization scheme in terms of exploration and exploitation. The details are explained in the following.

\subsection{Simplex Method}

The Nelder-Mead Simplex Algorithm method is classified as a heuristic optimization algorithm and direct research method, because the objective function is directly utilized to achieve the best optimal point without derivation. A simplex is a geometrical object produced by $(n+1)$ points $\left(x_{0}, \ldots, x_{n}\right)$ in $n$-dimension space [26,27]. For example, the triangle is a simplex in two-dimension space. The basic idea in the simplex method is to compare the value of the objective function at the $(n+1)$ vertices of a simplex and move the simplex gradually toward the optimum point through an iterative process. The vertices of a regular simplex (equilateral triangle in two dimensions) of 


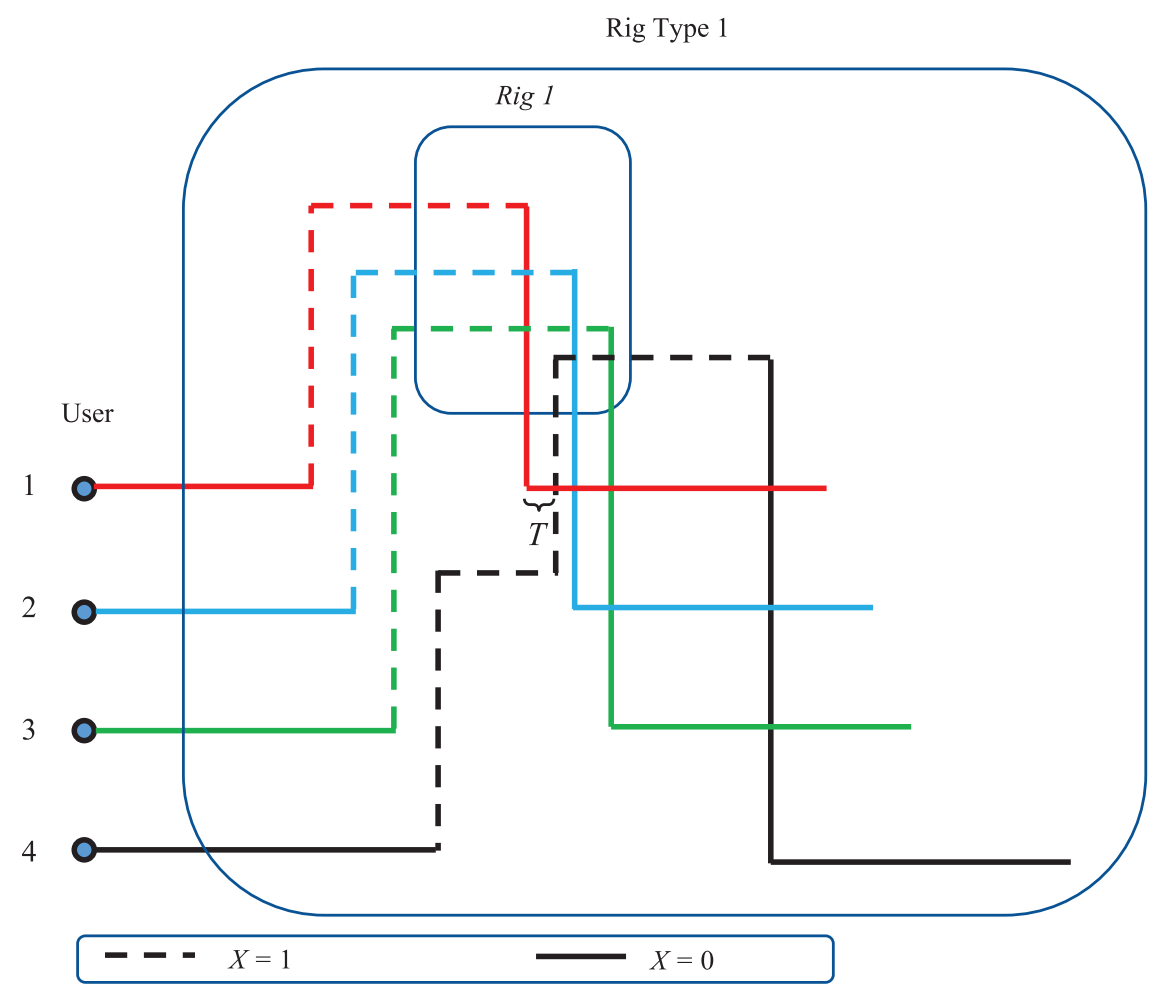

Fig. 1. Scheduling strategy.

size $a$, within $n$-dimensional space, are generated by Equation (2) [26],

$$
\mathbf{x}_{j}=\mathbf{x}_{0}+p \mathbf{u}_{j}+\sum_{\substack{s=1 \\ s \neq j}}^{n} q \mathbf{u}_{s},
$$

where $\mathbf{x}_{0}$ is the initial base point and $\mathbf{u}_{s}$ is the unit vector along the coordinate axis $s$ and also

$$
\begin{gathered}
p=\frac{a}{n \sqrt{2}}(\sqrt{n+1}+n-1), \\
q=\frac{a}{n \sqrt{2}}(\sqrt{n+1}-1) .
\end{gathered}
$$

For reaching the optimal solution, operational tools (reflection, contraction, and expansion) are utilized, deforming the simplex scheme geometrically (see Figure 2). After each transformation, the current worst vertex is swapped by a better one. Therefore, simplex gradually moves toward to the optimum point. The reflected, expanded and contracted points are given by: $\mathbf{x}_{r}, \mathbf{x}_{e}$, and $\mathbf{x}_{c}$, respectively:

$$
\begin{array}{cc}
\mathbf{x}_{r}=(1+\alpha) \overline{\mathbf{x}}_{0}-\alpha \mathbf{x}_{h} & \alpha>0, \\
\mathbf{x}_{e}=\gamma \overline{\mathbf{x}}_{r}+(1-\gamma) \overline{\mathbf{x}}_{0} & \gamma>1, \\
\mathbf{x}_{c}=\beta \mathbf{x}_{h}+(1-\beta) \overline{\mathbf{x}}_{0} & 0 \leq \beta \leq 1 .
\end{array}
$$

During these transformations, $\mathbf{x}_{0}$ is the centroid of all vertices $\mathbf{x}_{0}$ except $j=h$; where $h$ is the index of the worst point. The parameters $\alpha, \gamma$ and $\beta$ are called reflection, expansion, and contraction coefficients, respectively. 


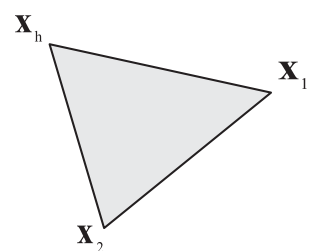

(a)

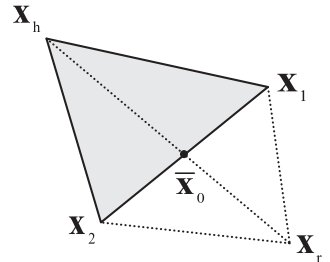

(b)

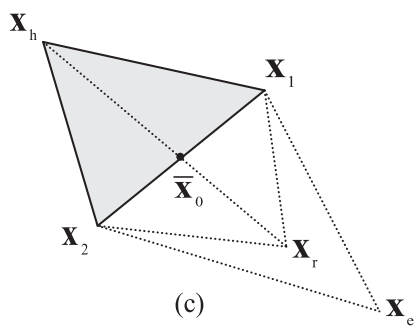

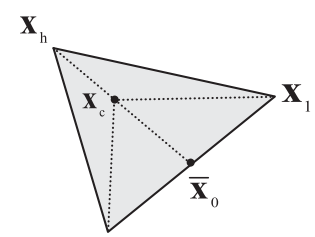

$\mathbf{x}_{2}$

(d)

Fig. 2. Available moves in the simplex method: (a) initial simplex; (b) reflection; (c) expansion; (d) contraction $[21,27]$.

Reflection is acting as reflecting the worst vertex, named high, concerning the centroid . If the reflected point is better than all other points, then expansion operates to expand the simplex in the reflection direction; otherwise, if it is at least better than the worst, then the algorithm performs the reflection with the new worst point again [26]. The contraction is operation because of which the worst point is at least as good as the reflected point.

\subsection{Nondominated Sorting Genetic Algorithm}

NSGA method is a direct optimization algorithm, like simplex method. This algorithm is known as a fast approach [28]. In this approach, to identify the solution of the first non-dominated front in a population of size $N_{\text {pop }}$, each solution can be compared with every other solution in the population to find out if it is dominated. This process is continued to find all members of the first non-dominated level in the population. This algorithm is utilized Selection, Crossover, and Mutation to find optimal points.

Generally, the algorithm begins with a feasible population of $N_{p o p}$ randomly selected initial solutions (individuals). The values of the objective functions are first calculated for each member of population. However, the individuals are not evaluated based on their actual objective function fitness, but it is considered based on the fitness values reflecting their nondominance properties and diversity. The algorithm handles the observability condition in the cost function by re-presenting the infeasible individuals using population classification, fitness sharing, crossover, mutation, and elitism.

Population Classification: The population is classified into subsets (fronts) of individuals sharing the same nondominance properties. The nondominated individuals presenting in the population are set to the first nondominated front $F^{1}$. These nondominated individuals are removed from the population, and a set of nondominated individuals in the rest of population $F^{2}$ is introduced. This process is continued until the entire population is classified into fronts. 
To give a preference to the individuals close to the Pareto-optimal front, dummy fitness $f_{d}^{r}$ is assigned to an individual in proportion to its front identifier $r$, which is formulated in Equation (8),

$$
f_{d}^{r}=\frac{T}{r}
$$

where the proportionality constant is the population size $N_{\text {pop }}$.

Fitness Sharing: It is likely that multiple solutions exits in the remote lab timetable problem. These solutions might have the same objective function values. To maintain the diversity of the population, the algorithm discourages the number of similar individuals by fitness sharing [20]. Fitness sharing allows multiple optimal solutions to coexist. The dummy fitness $f_{d}^{r}$, initially assigned to an individual $i$ in accordance to its front identifier $r$ is shared by the number of it "copies" $n_{i}$ present in the population (Equation (9)),

$$
f_{s h_{i}}^{r}=\frac{f_{d}^{r}}{n_{i}}
$$

where $f_{s h_{i}}^{r}$ represents the shared dummy fitness.

Crossover: The individuals in the entire population are reproduced according to their dummy fitness values (Equation (9)). The mating pool is formed by the stochastic remainder selection process [21], and the single point crossover with a probability across population is applied. Since crossover disrupts the feasibility of the solution, the majority of the solutions created by crossover are infeasible.

Mutation: A mutation operator is applied to the population with the probability. An individual is mutated to maintain the diversity in the candidate solutions. This operator adds a unit Gaussian distributed random value to the chosen individual.

Elitism: The algorithm preserves the solutions with good nondominated properties, called "elite," without modification from one generation to the other. After producing new individuals through crossover and mutation, the algorithm performs additional nondominated sorting of new and old individuals. Thereafter, it selects $N_{p o p}$ individuals from the combined pool as members of the new generation, starting from those with the highest dummy fitness.

Finally, the algorithm terminates when the number of generations $\left(N_{g e n}\right)$ reaches the predetermined maximum value.

\section{SIMPLEX NONDOMINATED SORTING GENETIC ALGORITHM}

This algorithm utilizes simplex optimization as exploration and NSGA as exploitation to find the optimal solution. The flowchart of SNSGA is shown in Figure 3. Also, the parameters of SNSGA are tuned and shown in Table 1.

\subsection{General Setting Out of the Algorithm}

SNSGA contains two heuristic algorithms, simplex optimization and NSGA, to reach the optimum global point. In this regard, simplex provides a wide range of variety across search space and acts as exploration; also, NSGA plays a vital role in exploiting the potential area, which likely consists of global optimization solutions. Moreover, simplex performs to generate the new population with enough diversity at each generation whereby selection, crossover, and mutation propagate vertices of the simplex. Therefore, simplex operations (reflection, expansion, and contraction) are carried out to deform and move simplex toward likelihood regions of the search space until a maximum number of iterations $\left(i_{\max }\right)$ is reached. SNSGA has ten parameters that must be set before the execution of the algorithm. 


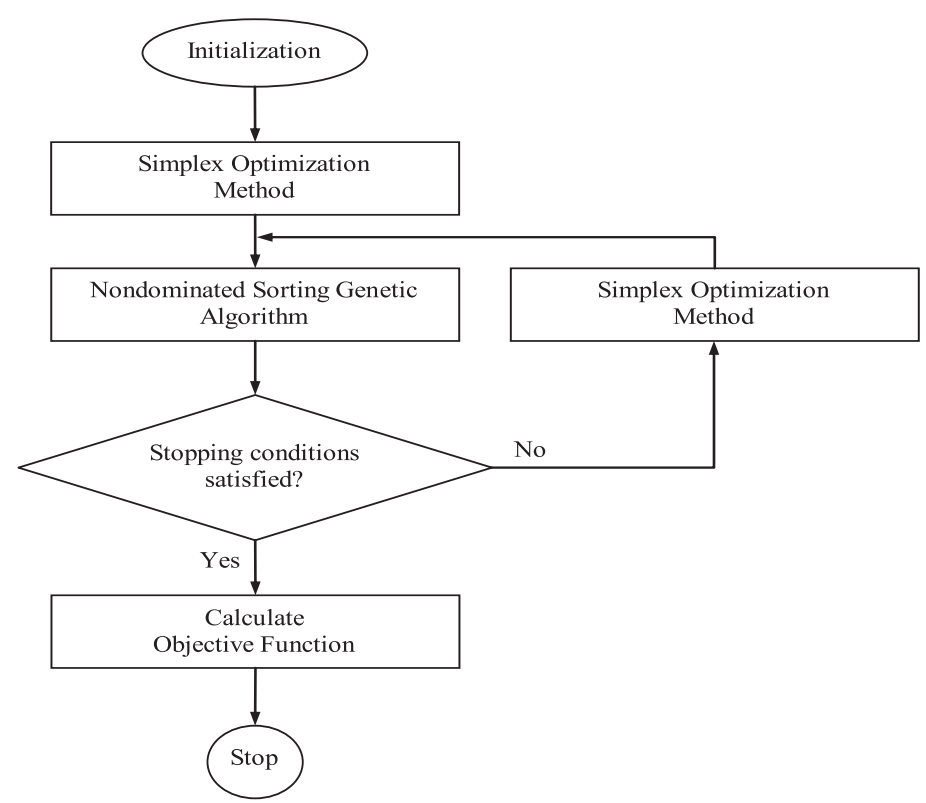

Fig. 3. Flowchart of SNSGA algorithm.

Table 1. Tuned Parameters of SNSGA

\begin{tabular}{ccc}
\hline Parameter & Description & Value \\
\hline$N_{\text {pop }}$ & Population Size & 30 \\
$N_{\text {gen }}$ & Maximum Generation & 60 \\
$R_{\text {crs }}$ & Crossover Ratio & 1.2 \\
$a_{\text {mut }}$ & Scale Parameter & 0.1 \\
$b_{\text {mut }}$ & Shrink Parameter & 0.5 \\
$a$ & Side of Simplex & 2 \\
$\alpha$ & Reflection Coefficient & 1 \\
$\gamma$ & Expansion Coefficient & 4 \\
$\beta$ & Contraction Coefficient & 0.2 \\
$i_{\text {max }}$ & Maximum Iteration & 30 \\
\hline
\end{tabular}

\subsection{Initial Population}

In this proposed scheme, the primary parent population is generated randomly with uniform distribution. Not only is a population of each generation sorted based on non-domination scheme but also each solution is assigned the fitness equal to its non-domination level. Furthermore, recombination based on binary tournament selection is carried out generating a first offspring having the same size as the parent population.

\subsection{Population Update}

In each generation, the population is sorted based on fast non-domination strategy. Simplex part of proposed algorithm organizes the new parent population. Simplex performs to generate the new 
Table 2. Computational Results of SNSGA for the Benchmarks

\begin{tabular}{lccc}
\hline $\begin{array}{l}\text { Test } \\
\text { Function }\end{array}$ & $\begin{array}{c}\text { Rate of } \\
\text { successful } \\
\text { minimization }\end{array}$ & $\begin{array}{c}\text { Average of objective } \\
\text { function numbers }\end{array}$ & $\begin{array}{c}\text { Average gap between the } \\
\text { best successful point and the } \\
\text { known global optimum }\end{array}$ \\
\hline $\mathrm{RC}$ & 100 & 109 & $1 e-6$ \\
$\mathrm{GP}$ & 100 & 124 & $8 e-5$ \\
$\mathrm{~B} 2$ & 100 & 94 & $1 e-6$ \\
$\mathrm{SH}$ & 100 & 206 & $5.5 e-5$ \\
$R_{2}$ & 100 & 189 & $4 e-6$ \\
$Z_{2}$ & 100 & 227 & $5 e-6$ \\
$H_{3,4}$ & 100 & 185 & $1.35 e-4$ \\
$S_{4,5}$ & 98 & 345 & $7 e-5$ \\
$R_{5}$ & 100 & 105 & $3 e-5$ \\
$R_{10}$ & 100 & 148 & $9 e-5$ \\
\hline
\end{tabular}

population with enough diversity, updating population through reflection, expansion and contraction operators for the next generation. Further, any individual that is generated by operational genetic tools is used as vertices of the simplex to achieve new population.

\subsection{Stop Condition}

Simplex part of the proposed algorithm performed in the inner loop terminates at the maximum number of iterations. NSGA part of the algorithm operated in outer loop satisfies termination by the maximum number of generation. Figure 3 illustrates that the parameters of SNSGA, listed in Table 1, must be set before running the algorithm. Then, initial populations that are appropriated for NSGA are produced by simplex method optimization. Therefore, the simplex method helps to increase exploration. Having created a population, NSGA carries out crossover and mutation to find the optimum global point. Afterward, the SNSGA will be stopped if stopping condition satisfies.

\section{NUMERICAL RESULTS}

SNSGA is applied on the benchmarks, listed in Reference [20]. According to Reference [20], SNSGA is executed 100 times to measure the rate of successful minimization, the average of the objective function evaluation numbers, and the average error on the objective function. Once either one of the termination criteria is first reached, the algorithm stops and returns the coordinates of a final point as well as the final optimal objective function value $\left(F O B J_{A L G}\right.$ (algorithm)). Analytical minimum objective value $\left(F O B J_{A N A L}\right)$ is compared with $F O B J_{A L G}$, and thus the solution is said to be "successful" if the following inequality holds:

$$
\left|F O B J_{A L G}-F O B J_{A N A L}\right|<10^{-4}\left|F O B J_{I N I T}\right|+10^{-6},
$$

where $F O B J_{I N I T}$ is an average of the objective function.

The average of the objective function evaluation numbers is only accounted for the "successful minimization." The average error is defined as the average of FOBJ deviation between the best successful point and the known global optimum, where only the "successful minimization" achieved by the algorithm. The results of SNGSA tests performed over ten benchmarks are shown in Table 2. 
Table 3. Average Number of Objective Function Evaluation

\begin{tabular}{lcccccccc}
\hline Algorithm & $\mathrm{RC}$ & $\mathrm{GP}$ & $B_{2}$ & $\mathrm{SH}$ & $R_{2}$ & $Z_{2}$ & $H_{3,4}$ & $S_{4,5}$ \\
\hline CHA [29] & 295 & 259 & 132 & 345 & 459 & 215 & 492 & 598 \\
ECTS [30] & 245 & 231 & 210 & 370 & 480 & 195 & 548 & 825 \\
CGA [31] & 620 & 410 & 320 & 575 & 960 & 620 & 582 & 610 \\
ESA [32] & - & 783 & - & - & 796 & 15820 & 698 & 1137 \\
CRTS min [33] & 41 & 171 & - & - & - & - & 609 & 664 \\
CRTSave [33] & 38 & 248 & - & - & - & - & 513 & 812 \\
TS [34] & 492 & 486 & - & 727 & - & - & 508 & - \\
INTEROPT [35] & 4172 & 6375 & - & - & - & - & 1113 & 3700 \\
NM-GA [36] & 356 & 422 & 529 & 1009 & 738 & 339 & 688 & 2366 \\
NM-PSO [36] & 230 & 304 & 325 & 753 & 440 & 186 & 436 & 850 \\
\hline SNSGA & 109 & 124 & 94 & 206 & 189 & 227 & 185 & 345 \\
\hline
\end{tabular}

The performance of SNGSA is compared with other algorithms such as Continuous Hybrid Algorithm (CHA) [29], Enhanced Continuous Tabu Search (ECTS) [30], Continuous Genetic Algorithm (CGA) [31], Enhanced Simulated Annealing (ESA) [32], Continuous Reactive Tabu Search minimum (CRTS min) [33], Continuous Reactive Tabu Search average (CRTS ave) [33], Tabu search (TS) [34], INTEROPT [35], Hybrid Nelder-Mead simplex method and Genetic Algorithm (NM-GA) [36], and Hybrid Nelder-Mead simplex method and Particle Swarm Optimization (NM-PSO) [36]. Table 3 illustrates the average numbers of function evaluation over 100 simulation runs for each benchmark and of the optimization algorithm.

The numerical results demonstrate that SNSGA has a significant performance improvement in comparison with other hybrid heuristic algorithms. Overall, there is a considerable decrease in the average number of objective function evaluation for almost all benchmarks except for $Z_{2}$. Some of the test functions listed in Table 3 are utilized to describe the performance of the proposed algorithm in finding of optimum global point efficiently. Note that the objective function is normalized. The normalized objective functions are formulated as follows:

$$
N O F=\frac{O F-\min (O F)}{\max (O F)-\min (O F)}
$$

where NOF is the normalized objective function in each iteration. OF is the real value of the objective function, while $\max (O F)$ and $\min (O F)$ are maximum and minimum values of the objective functions, respectively, throughout the iteration.

Figure 4 represents the convergence performance of SNSGA starting from an initial random point versus the number of iteration. As seen, the SNSGA has precisely reached to global optimums during the iterations. Thus, SNSGA is competitive and even better than other meta-heuristic optimization schemes.

SNSGA is utilized to reach the optimum scheme of a scheduling problem for remote labs. Figure 5 shows the results of the optimization for the scheduling problem. Figure 5 represents the periodical form of timetable problem. This periodical item is completely depended on the number of requests using the specific rig type. When the scheduling process starts with one user, the value of the objective function begins from 2 decreasing gradually in zero based on the request and capacity. Therefore, this trend is periodically occurred to make room for those who cannot use rigs. 


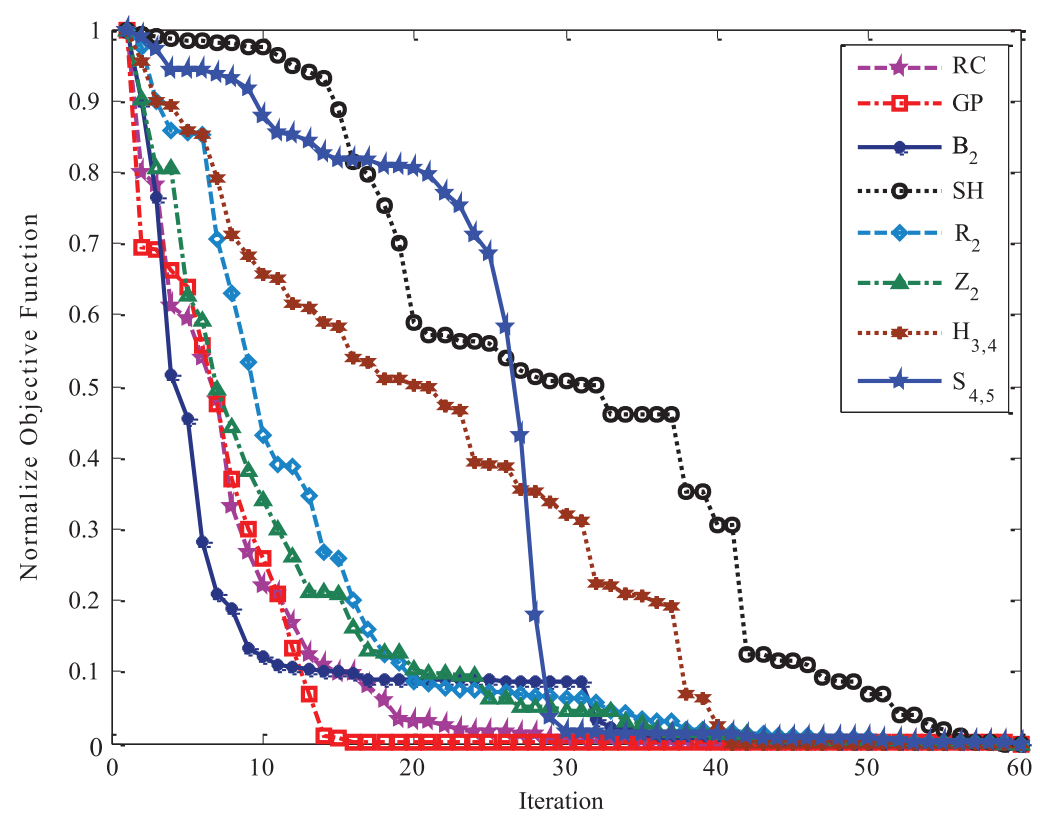

Fig. 4. Objective function value of the some benchmarks versus iteration number.

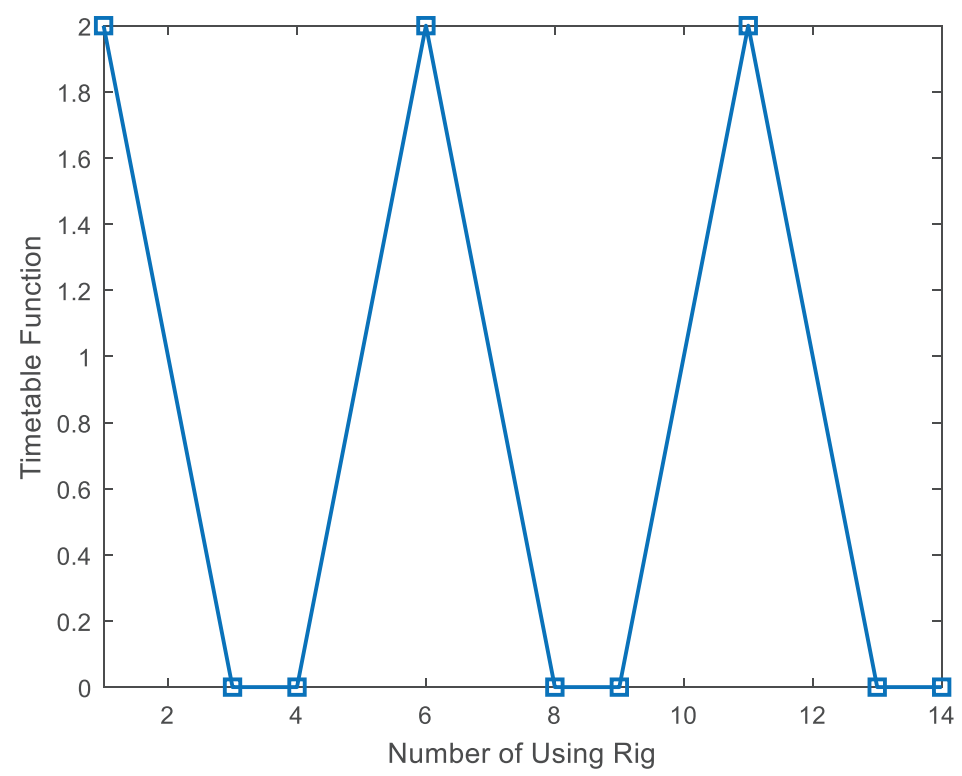

Fig. 5. Minimization of the objective function for timetable scheduling mode.

\section{CONCLUSION}

A new hybrid heuristic optimization algorithm was proposed to respond to the demand for having access to the remote laboratories. The proposed hybrid algorithm formulates the scheduling problem as a nonlinear optimization problem. The hybridization of Nelder-Mead simplex and nondominated sorting genetic algorithm reached a good compromise between time-consuming and 
accuracy. The numerical results show that the proposed algorithm has a competitive performance in solving scheduling problems.

\section{REFERENCES}

[1] Seid Miad Zandavi, Zexi Hu, Yuk Ying Chung, and Ali Anaissi. 2019. Augmented reality vision improving educational learning. Aust. J. Intell. Inf. Process. Syst. 15, 3 (2019), 49-58.

[2] Mahmoud Abdulwahed, Zoltan K. Nagy, Richard E. Blanchard, et al. 2008. Beyond the classroom walls: Remote labs, authentic experimentation with theory lectures. In Proceedings of the 19th Annual Conference of the Australasian Association for Engineering Education: To Industry and Beyond. Institution of Engineers, Australia, 435.

[3] V. Judson Harward, Jesus A. Del Alamo, Steven R. Lerman, Philip H. Bailey, Joel Carpenter, Kimberley DeLong, Chris Felknor, James Hardison, Bryant Harrison, Imad Jabbour, et al. 2008. The ilab shared architecture: A web services infrastructure to build communities of internet accessible laboratories. Proc. IEEE 96, 6 (2008), 931-950.

[4] David Lowe, Stephen Conlon, Steve Murray, Lothar Weber, Michel De La Villefromoy, Euan Lindsay, Andrew Nafalski, Warren Nageswaran, and Tee Tang. 2012. LabShare: Towards cross-institutional laboratory sharing. In Internet Accessible Remote Laboratories: Scalable E-learning Tools for Engineering and Science Disciplines. IGI Global, 453-467.

[5] Jing Ma and Jeffrey V. Nickerson. 2006. Hands-on, simulated, and remote laboratories: A comparative literature review. ACM Comput. Surveys 38, 3 (2006), 7.

[6] Christophe Gravier, Jacques Fayolle, Bernard Bayard, Mikael Ates, and Jeremy Lardon. 2008. State of the art about remote laboratories paradigms-foundations of ongoing mutations. Int. f. Online Eng. 4, 1 (2008), http-www.

[7] Nawat Nuntasen and Supachate Innet. 2007. Application of genetic algorithm for solving university timetabling problems: A case study of Thai universities. UTCC Engineering Research Papers.

[8] Clemens Nothegger, Alfred Mayer, Andreas Chwatal, and Günther R. Raidl. 2012. Solving the post enrolment course timetabling problem by ant colony optimization. Ann. Oper. Res. 194, 1 (2012), 325-339.

[9] Der-Fang Shiau. 2011. A hybrid particle swarm optimization for a university course scheduling problem with flexible preferences. Expert Syst. Appl. 38, 1 (2011), 235-248.

[10] Ioannis X. Tassopoulos and Grigorios N. Beligiannis. 2012. A hybrid particle swarm optimization-based algorithm for high school timetabling problems. Appl. Soft Comput. 12, 11 (2012), 3472-3489.

[11] Leena N. Ahmed, Ender Özcan, and Ahmed Kheiri. 2015. Solving high school timetabling problems worldwide using selection hyper-heuristics. Expert Syst. Appl. 42, 13 (2015), 5463-5471.

[12] Sener Akpinar. 2016. Hybrid large neighbourhood search algorithm for capacitated vehicle routing problem. Expert Syst. Appl. 61 (2016), 28-38.

[13] Jian Li, Panos M. Pardalos, Hao Sun, Jun Pei, and Yong Zhang. 2015. Iterated local search embedded adaptive neighborhood selection approach for the multi-depot vehicle routing problem with simultaneous deliveries and pickups. Expert Syst. Appl. 42, 7 (2015), 3551-3561.

[14] Amin Jamili, Mohammad Ali Shafia, Seyed Jafar Sadjadi, and Reza Tavakkoli-Moghaddam. 2012. Solving a periodic single-track train timetabling problem by an efficient hybrid algorithm. Eng. Appl. Artific. Intell. 25, 4 (2012), 793-800.

[15] Safaai Deris, Sigeru Omatu, Hiroshi Ohta, and Puteh Saad. 1999. Incorporating constraint propagation in genetic algorithm for university timetable planning. Eng. Appl. Artific. Intell. 12, 3 (1999), 241-253.

[16] Seid Miad Zandavi and Vera Chung. 2019. State estimation of nonlinear dynamic system using novel heuristic filter based on genetic algorithm. Soft Comput. 23, 14 (2019), 5559-5570.

[17] Shu-Kai S Fan, Yun-chia Liang, and Erwie Zahara. 2004. Hybrid simplex search and particle swarm optimization for the global optimization of multimodal functions. Eng. Optimiz. 36, 4 (2004), 401-418.

[18] Shu-Kai S Fan, Yun-Chia Liang, and Erwie Zahara. 2006. A genetic algorithm and a particle swarm optimizer hybridized with Nelder-Mead simplex search. Comput. Industr. Eng. 50, 4 (2006), 401-425.

[19] Seid Miad Zandavi. 2017. Surface-to-air missile path planning using genetic and PSO algorithms. f. Theoret. Appl. Mech. 55, 3 (2017), 801-812.

[20] Seid Miad Zandavi, Vera Yuk Ying Chung, and Ali Anaissi. 2019. Stochastic dual simplex algorithm: A novel heuristic optimization algorithm. IEEE Trans. Cybernet. (2019), 1-10.

[21] S. H. Pourtakdoust and S. M. Zandavi. 2016. A hybrid simplex non-dominated sorting genetic algorithm for multiobjective optimization. Int. F. Swarm Intell. Evolution. Comput. 5, 3 (2016), 1-11.

[22] Seid Miad Zandavi and Seid H. Pourtakdoust. 2018. Multidisciplinary design of a guided flying vehicle using simplex nondominated sorting genetic algorithm II. Struct. Multidisc. Optimiz. 57, 2 (2018), 705-720.

[23] Natércia Lima, Clara Viegas, and Francisco José García-Peñalvo. 2019. Different didactical approaches using a remote lab: Identification of impact factors. IEEE Revista Iberoamericana de Tecnologias del Aprendizaje 14, 3 (2019), 76-86. 
[24] Clara Viegas, Ana Pavani, Natércia Lima, Arcelina Marques, Isabel Pozzo, Elsa Dobboletta, Vanessa Atencia, Daniel Barreto, Felipe Calliari, André Fidalgo, et al. 2018. Impact of a remote lab on teaching practices and student learning. Comput. Edu. 126 (2018), 201-216.

[25] S. M. Zandavi and V. Chung. 2018. Augmented reality for remote laboratory improving educational learning: Using elevated particle swarm optimization in object tracking scheme. In Proceedings of the International foint Conference on Neural Networks (IFCNN'18). 1-6.

[26] Singiresu S. Rao. 2009. Engineering Optimization: Theory and Practice. John Wiley \& Sons.

[27] Hadi Nobahari, Seid Miad Zandavi, and Hamed Mohammadkarimi. 2016. Simplex filter: A novel heuristic filter for nonlinear systems state estimation. Appl. Soft Comput. 49 (2016), 474-484.

[28] Nidamarthi Srinivas and Kalyanmoy Deb. 1994. Muiltiobjective optimization using nondominated sorting in genetic algorithms. Evolution. Comput. 2, 3 (1994), 221-248.

[29] Rachid Chelouah and Patrick Siarry. 2003. Genetic and nelder-mead algorithms hybridized for a more accurate global optimization of continuous multiminima functions. Eur. F. Operation. Res. 148, 2 (2003), 335-348.

[30] Rachid Chelouah and Patrick Siarry. 2000. Tabu search applied to global optimization. Eur. f. Operation. Res. 123, 2 (2000), 256-270.

[31] Rachid Chelouah and Patrick Siarry. 2000. A continuous genetic algorithm designed for the global optimization of multimodal functions. F. Heurist. 6, 2 (2000), 191-213.

[32] Pyari Mohan Pradhan and Ganapati Panda. 2012. Solving multiobjective problems using cat swarm optimization. Expert Syst. Appl. 39, 3 (2012), 2956-2964.

[33] Jesus Moises Osorio Velazquez, Carlos A. Coello Coello, and Alfredo Arias-Montano. 2014. Multi-objective compact differential evolution. In Proceedings of the IEEE Symposium on Differential Evolution (SDE'14). IEEE, 1-8.

[34] Hossein Hemmatian, Abdolhossein Fereidoon, and Ehsanolah Assareh. 2014. Optimization of hybrid laminated composites using the multi-objective gravitational search algorithm (MOGSA). Eng. Optimiz. 46, 9 (2014), 1169-1182.

[35] Xiangui Shi and Dekui Kong. 2015. A multi-objective ant colony optimization algorithm based on elitist selection strategy.Metallurg. Mining Industry6 (2015).

[36] Emrah Hancer, Bing Xue, Mengjie Zhang, Dervis Karaboga, and Bahriye Akay. 2015. A multi-objective artificial bee colony approach to feature selection using fuzzy mutual information. In Proceedings of the IEEE Congress on Evolutionary Computation (CEC'15). IEEE, 2420-2427.

Received September 2019; revised August 2020; accepted November 2020 